\title{
NIST Syndromic Surveillance Test Suite 2015 Edition
}

\author{
Sheryl L. Taylor* and Robert Snelick \\ National Institute of Standards and Technology, Gaithersburg, MD, USA
}

\section{Objective}

The NIST Syndromic Surveillance Test Suite for 2015 Edition ONC certification testing was published in February 2016. Key information related to the purpose, development, and use of this conformance test tool is provided via snapshots on a poster.

\section{Introduction}

Details about the ONC 2015 Edition certification criteria for Syndromic Surveillance and the related NIST Test Suite were explained previously. We now provide an overview and key information regarding updates to the Test Suite and how it is designed to be used.

\section{Methods}

Snapshots are provided on a poster and are used by the presenter to explain the steps involved in developing the NIST Syndromic Surveillance Test Suite 2015 Edition, to show key features of and updates to the Test Suite, and to illustrate the relationship of the Test Suite to various releases of the PHIN Messaging Guide for Syndromic Surveillance.

\section{Results}

The NIST Syndromic Surveillance Test Suite for 2015 Edition ONC certification testing was published in February 2016. As the target stakeholders began using it and providing feedback, this tool and associated documentation were updated. The Test Suite is being used by test labs for ONC certification testing of health information technologies, by developers in preparation for certification testing, and ultimately by public health jurisdictions for on-boarding of provider organizations that need to submit surveillance data.

\section{Conclusions}

Automated conformance test tools enable validation of health information technologies' ability to support the requirements published in the PHIN Messaging Guide for Syndromic Surveillance. Having this standard and the means to validate conformance helps drive the industry toward the level of interoperability needed to promote efficient reporting and utilization of syndrome-based public health surveillance information.

\section{Keywords}

conformance testing; test tool; interoperability standards

\section{Acknowledgments}

The NIST Syndromic Surveillance Test Suite was developed in collaboration with subject matter experts at The Centers for Disease Control and Prevention.

\section{*Sheryl L. Taylor}

E-mail: sheryl.taylor@nist.gov 